\title{
Estimación de los rangos térmicos asociados con la distribución de Sardinops sagax caeruleus, con base en su preferencia térmica
}

Estimation of the thermal ranges associated with the distribution of Sardinops sagax caeruleus, based on its thermal preference

Marcel Martínez-Porchas ${ }^{1}$ y Mónica Hernández-Rodriguez ${ }^{2,3}$

\author{
${ }^{1}$ Centro de Investigación en Alimentación y Desarrollo (CIAD) A.C., Km 0,6 Carretera a La Victoria ZC 83304, Hermosillo, \\ Sonora, México \\ ${ }^{2}$ Centro de Investigación Científica y Educación Superior de Ensenada (CICESE), Km. 107, Carretera Tijuana-Ensenada, Baja \\ California, México.mhernand@cicese.mx \\ ${ }^{3}$ Centro de Investigación Científica y Educación Superior de Ensenada (CICESE), Departamento de Acuicultura, P.O. Box: \\ 434844, San Diego, CA. 92143-4844, Estados Unidos
}

\begin{abstract}
The Pacific sardine (Sardinops sagax caeruleus) from Ensenada, Mexico, was exposed to two thermal regimes, cold $\left(\mathrm{CR}: 13-18^{\circ} \mathrm{C}\right)$ and warm $\left(\mathrm{WR}: 18-23^{\circ} \mathrm{C}\right)$ for 20 days. The thermal preference was studied using a gradient of temperatures, recording the preferred and avoided sites by the sardine. No significant differences among acclimation thermal regimes were observed for thermal preference. However, there was some difference for avoided temperatures showed by these two groups of sardine; $13.3-19.3^{\circ} \mathrm{C}$ was for $\mathrm{CR}$ and $16.9-22.3^{\circ} \mathrm{C}$ for WR. These results suggest that the studied sardine are able to survive within a thermal environment from 13.3 to $22.3^{\circ} \mathrm{C}$, which shifts in the area delimited by San Francisco and Magdalena Bay.
\end{abstract}

Key words: Pacific sardine, distribution, thermal behavior, migration

\section{INTRODUCCIÓN}

La importancia ecológica y económica de la sardina del Pacífico, Sardinops sagax caeruleus, ha provocado que estudios científicos se enfoquen en su dinámica poblacional con el objetivo de elaborar adecuados esquemas de manejo pesquero para la preservación de este recurso. No obstante, es difícil entender las respuestas biológicas de la especie ante cambios en el ecosistema, siendo en algunas ocasiones impredecible. Uno de los temas más estudiados ha sido su distribución, aunque hasta el momento no ha sido posible establecer límites geográficos específicos, lo cual también podría atribuirse a la falta de un sistema de monitoreo permanente. Una de las teorías más aceptadas, es la existencia de diferentes sub-poblaciones de sardina a lo largo del Pacífico norte; Felix-Uraga et al. (2004) establecieron un modelo de distribución en base a datos de captura y temperatura superficial a lo largo de 20 años, concluyendo que existen tres sub-poblaciones (metapoblaciones) separadas por barreras térmicas y espaciotemporales. Así, se sugirió la existencia de una sub-población 'fría' que habita en la zona de California $\left(36-32^{\circ} \mathrm{N} ; 13-17^{\circ} \mathrm{C}\right)$, una sub-población 'templada' a lo largo de la Península de Baja California $\left(31-26^{\circ} \mathrm{N} ; 17-22^{\circ} \mathrm{C}\right)$; y una sub-población 'cálida' al sur de la península de Baja California $\left(24-22^{\circ} \mathrm{N}\right.$; $\left.>22^{\circ} \mathrm{C}\right)$. Sin embargo, aunque el estudio establece límites térmicos para esta especie, no precisa su comportamiento térmico, lo cual permitiría conocer en forma más amplia su posible distribución y con ello mejorar programas de protección y pesca.

En particular, la sub-población templada quizás sea la más importante debido a su contribución a la industria pesquera. Durante el verano, estos peces pueden experimentar fluctuaciones térmicas diarias que oscilan entre 18 y $23^{\circ} \mathrm{C}$, mientras que en invierno la fluctuación térmica es de alrededor de 13 y $18^{\circ} \mathrm{C}$ (Martínez-Porchas 2009). Aunque la sardina puede migrar verticalmente hacia otras temperaturas, estas fluctuaciones pueden llegar a tener un efecto sobre su comportamiento térmico, lo que también podría reflejarse en su distribución geográfica. El objetivo de este trabajo fue estudiar en laboratorio la preferencia térmica de la sub-población templada de $S$. sagax caeruleus y estimar la zona geográfica que contiene la temperatura óptima para esta sub-población.

\section{Material y Métodos}

Se capturaron sardinas de la Bahía de Ensenada, México $\left(31^{\circ} \mathrm{N}, 117^{\circ} \mathrm{W}\right)$ mediante un diseño de muestreo simple, 
durante el mes de septiembre (2008) y se colocaron en bolsas plásticas con agua de mar a $20^{\circ} \mathrm{C}$ y oxígeno disuelto (OD) $\geq 6 \mathrm{mg} \cdot \mathrm{L}^{-1}$. Los ejemplares fueron transportados al laboratorio de peces marinos del 'Centro de Investigación Científica y de Educación Superior de Ensenada (CICESE)'. Las sardinas tenían una talla de $19-21 \mathrm{~cm}$, peso de 67-74 g y una edad de 2,5-3 años.

Las sardinas fueron colocadas en tanques con capacidad de $5000 \mathrm{~L}$ y se aclimataron a condiciones de laboratorio durante 15 días (temperatura: $20 \pm 0,2^{\circ} \mathrm{C}$; OD: $5,79 \pm 0,3$ $\mathrm{mg} \cdot \mathrm{L}^{-1}$; fotoperiodo $12: 12$; recambio de agua $300 \% \cdot$ día $^{-1}$; pH: 7,9-8,0; $\mathrm{NH}_{3}-\mathrm{NH}_{4}: 0,2 \pm 0,1 \mathrm{mg} \cdot \mathrm{L}^{-1} \mathrm{y}$ alimentación ad libitum). Posteriormente las sardinas fueron expuestas a dos regímenes térmicos tomando en cuenta la fluctuación térmica diaria en aguas de Ensenada y lugares aledaños durante invierno y verano a una profundidad de $20 \mathrm{~m}$ (com. pers. Dra. Ladah ${ }^{1}$ ); la profundidad fue estimada considerando reportes de la distribución preferencial de estos organismos, que sería en la superficie, alrededor de 20-30 m de profundidad (Emmett et al. 2005, Santos et al. 2006). La aclimatación de los especímenes, consistió en dos regímenes; uno cálido (RC) que tuvo una fluctuación térmica de 18 a $23^{\circ} \mathrm{C}$, permaneciendo a $18^{\circ} \mathrm{C}$ durante 10 $\mathrm{h}$, aumentando hasta $23^{\circ} \mathrm{C}$ por $5 \mathrm{~h}$, para permanecer constante por otras $4 \mathrm{~h}$ y finalmente descenderla a $18^{\circ} \mathrm{C}$ en $5 \mathrm{~h}$ (10-5-4-5), y el otro régimen fue uno frío (RF) cuya temperatura fluctuó de 13 a $18^{\circ} \mathrm{C}$, con tiempos de variaciones similares a los del régimen anterior. Para cada régimen térmico se implementaron 4 tanques redondos de 320 L, cada uno con 40 sardinas. Los parámetros fisicoquímicos del agua fueron similares y el tiempo de exposición fue de 20 días.

Después de la exposición a los regímenes térmicos, se estudió la preferencia térmica de las sardinas aclimatadas en ambos regímenes térmicos. Para esto, se utilizaron dos gradientes térmicos $\left(10-25^{\circ} \mathrm{C}\right)$ de 3,3 $\mathrm{m}$ de longitud con 15 cámaras virtuales cada uno. En un extremo del gradiente se aumentó la temperatura del agua con dos calentadores de titanio de 1000 Watt conectados a un controlador térmico (Chrontrol XT, Chrontrol ${ }^{\circledR}$ Corp.), mientras que en el otro extremo la temperatura fue disminuida por medio de un enfriador (Neslab ${ }^{\circledR}$ HX-100). Para evitar la estratificación, se colocó una manguera de aireación a lo largo de todo el gradiente (Bückle et al. 2003). En cada gradiente se simularon condiciones día-noche (12:12 h), en donde la intensidad de luz disminuía de manera constante hasta llegar a condiciones de obscuridad en 30 $\min$.

${ }^{1}$ Dra. Lydia Ladah, CICESE, México.
Para estudiar la preferencia térmica, se colocaron 4 sardinas dentro de cada gradiente en la cámara que tenía una temperatura similar a la de los tanques experimentales; después de 30 min se registró cada hora la temperatura de la cámara en la que se encontraba cada sardina durante 24 h, según el criterio de Hernández-Rodríguez et al. (2002). El promedio general de las mediciones realizadas en $24 \mathrm{~h}$ se consideró como el valor puntual de la preferencia térmica. También, fue observado el comportamiento natatorio de la sardinas y se registró la temperatura de las cámaras que visitaban en ambos extremos térmicos con el objetivo de determinar el intervalo térmico en el que cual se encontraba cada grupo de sardinas. Con base en estos datos, se estableció un intervalo de preferencia térmica, indicativo de que la sardina prefería permanecer dentro de dicho intervalo y evitaba introducirse en cámaras con temperaturas inferiores o superiores.

Los datos de evitación y preferencia térmica se analizaron por medio de un análisis de varianza de medidas repetidas (Zar 1996), tomando en cuenta los regímenes de aclimatación como los tratamientos y cada tanque experimental como repetición. Se consideró un nivel de significancia de $\alpha=0,05$ y se utilizaron los valores puntuales para establecer el intervalo térmico de distribución.

\section{Resultados y Discusión}

Las sardinas de ambos tratamientos (cálido y frío) mostraron una preferencia térmica estadísticamente similar a pesar del tipo de aclimatación, con valores promedio de $18,1 \pm 1,1^{\circ} \mathrm{C}$ para las sardinas expuestas al régimen frío y $19,0 \pm 1,4^{\circ} \mathrm{C}$ para las expuestas al régimen cálido $\left(\mathrm{F}_{(1,63)}\right)$ $143,5, P=0,08)$. No se registraron diferencias significativas entre la preferencia térmica en condiciones de luz y obscuridad $\left(\mathrm{F}_{(1,63)}=15,22, P>0,35\right)$. La preferencia térmica de las sardinas concuerda con el trabajo previo de Felix-Uraga et al. (2004), quienes demostraron que los mayores volúmenes de captura de esta sub-población se obtienen en aguas con temperatura superficial entre los 18 y $20^{\circ} \mathrm{C}$. Martínez-Porchas (2009) reportó que la sardina expuesta a $18^{\circ} \mathrm{C}$ no mostraba síntomas de estrés al evaluar distintos parámetros sanguíneos.

En cuanto a la evitación térmica, el tipo de aclimatación tuvo un efecto significativo sobre este parámetro (Fig. 1) $\left(\mathrm{F}_{(1,63)}=107,3, P<0,001\right)$. Las temperaturas de evitación de las sardinas expuestas al RF fueron de $13,3 \pm 1,5^{\circ} \mathrm{C}$ para el extremo inferior y $19,2 \pm$ $0,9^{\circ} \mathrm{C}$ para el extremo superior; mientras que las expuestas 


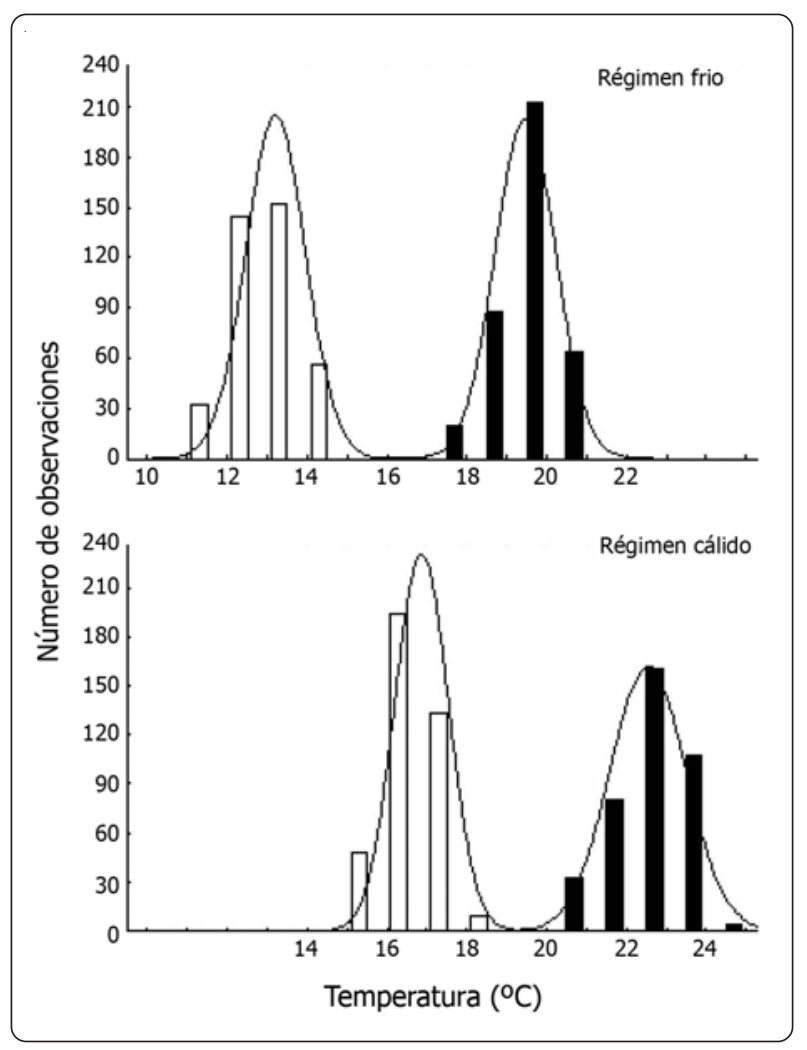

Figura 1. Distribución de frecuencia de temperaturas de evitación inferior (barras blancas) y superior (negras) de Sardinops sagax caeruleus expuesta un régimen frío $\left(13-18^{\circ} \mathrm{C}\right)$ y uno cálido $\left(18-23^{\circ} \mathrm{C}\right) /$ Frequency distribution of lower (white bars) and higher (black bars) avoided temperatures of Sardinops sagax caeruleus exposed to a cold $\left(13-18^{\circ} \mathrm{C}\right)$ and a warm regime $\left(18-23^{\circ} \mathrm{C}\right)$

al RC mostraron una evitación térmica desde $16,9 \pm 0,8^{\circ} \mathrm{C}$ hasta $22,3 \pm 1,3^{\circ} \mathrm{C}$. Es decir, que el intervalo de evitación térmica se desplazó en alrededor de $3^{\circ} \mathrm{C}$ cuando las sardinas fueron aclimatadas a una temperatura superior. Estos resultados muestran que la sardina posee plasticidad térmica y puede modificar sus temperaturas de evitación cuando son aclimatadas a diferentes temperaturas, lo que refuerza la hipótesis de que la sardina del Pacífico se comporta como un organismo euritermo (Takasuka 2007). En base a este intervalo térmico $\left(13,3-22,3^{\circ} \mathrm{C}\right)$, puede establecerse una zona geográfica que comúnmente registre estos valores para cada trimestre del año, tomando en cuenta la temperatura promedio mensual de distintas localidades a lo largo del Pacífico Norte en 20 m de profundidad de la columna de agua según el reporte de Lynn et al. (1982). De esta manera, se obtiene una zona térmica óptima de miles de kilómetros. Esta zona abarcaría desde Bahía Magdalena, Baja California-Sur (México, $24^{\circ} 36^{\prime} \mathrm{N}, 112^{\circ} 12^{\prime} \mathrm{W}$ ) hasta San Pedro, California (Estados
Unidos, $33^{\circ} 42^{\prime} \mathrm{N}, 118^{\circ} 27^{\prime} \mathrm{W}$ ) durante el primer trimestre del año (> $1100 \mathrm{~km}$ ); posteriormente para el tercer trimestre esta zona ya se ha desplazado hacia el norte más de 500 km, abarcando las localidades de Isla Cedros BC (México, $28^{\circ} 05^{\prime} \mathrm{N}, 115^{\circ} 30^{\prime} \mathrm{W}$ ) hasta la bahía de San Francisco (Estados Unidos, $37^{\circ} 45^{\prime} \mathrm{N}, 112^{\circ} 40^{\prime} \mathrm{W}$; > $1200 \mathrm{~km}$ ). Esto podría explicar la migración que ocurre estacionalmente e incluso cuando suceden fenómenos climatológicos como el Niño o la Niña; de hecho, el desplazamiento de la zona térmica $\left(13,3-22,3^{\circ} \mathrm{C}\right)$ a lo largo del año encontrado en los reportes de Lynn et al. (1982) concuerda con el movimiento migratorio de la sub-población templada de sardina propuesto por Felix-Uraga et al. (2004). En el caso de fenómenos climatológicos, esta zona térmica puede desplazarse 'anormalmente'; por ejemplo, en el Golfo de California se ha observado que durante períodos de la Niña las sardinas migran hacia lugares sureños que normalmente no visitarían (Lluch-Belda et al. 1986), posiblemente debido a que su zona térmica optima se desplaza más de lo normal hacia latitudes menores.

Los resultados también mostraron que las sardinas estudiadas migraron a temperaturas bajas $\left(13,3^{\circ} \mathrm{C}\right)$ cuando fueron aclimatadas al RF, temperaturas comunes en lugares más norteños, donde habitaría la sub-población fría. Martínez-Porchas et al. (2009) demostraron que la sardina del Pacífico capturada en Ensenada posee una elevada tolerancia a bajas temperaturas $\left(<10^{\circ} \mathrm{C}\right)$. Por otro lado, los resultados sugieren que esta sub-población no es capaz de introducirse en aguas con temperaturas superiores a los $24^{\circ} \mathrm{C}$ tal como las encontradas en Bahía Magdalena o el Golfo de California en donde también se ha documentado la presencia de sardina, la cual pertenece a la sub-población cálida (Lluch-Belda et al. 1986, Félix-Uraga et al. 2004). En el estudio realizado por Martínez-Porchas et al. (2009) se observó que la sardina de Ensenada presentaba una temperatura letal incipiente superior de alrededor de $25^{\circ} \mathrm{C}$, lo que podría explicar porque estos peces evitaban introducirse en temperaturas mayores a $23^{\circ} \mathrm{C}$.

Aunque los resultados demuestran el efecto de la temperatura sobre el comportamiento térmico de la sardina y su posible distribución, existen también otros factores (e.g., salinidad, oxígeno disuelto, surgencias, intensidad de luz y disponibilidad de alimento) que pueden afectar la distribución de las especies del género Sardinops y otros pelágicos (Butler et al. 1993, Bertrand et al. 2004, Emmett et al. 2005, Martínez-Porchas 2009). La temperatura es un factor importante para la fisiología y distribución de las sardinas; no obstante, otros estudios han revelado que después de la temperatura, la presencia de alimento, los 
períodos de reproducción y posiblemente la longitud corporal pueden ser factores determinantes en la distribución de esta especie (Lluch-Belda et al. 1991).

Finalmente, considerando solamente el factor de temperatura, puede concluirse que la sardina adulta capturada en Ensenada tiene capacidad para sobrevivir en temperaturas desde los 13,3 hasta los $22,3^{\circ} \mathrm{C}$.

\section{Agradecimientos}

Agradecemos al Consejo Nacional de Ciencia y Tecnología (CONACYT) por el soporte financiero a esta investigación (P 46060-Z). También se agradece a los técnicos Luis Murillo, Francisco Valenzuela, Carlos Carballo, Norberto Flores y José Espinoza por su apoyo en el transporte y manejo de organismos vivos.

\section{LITERATURA CITADA}

Bertrand A, M Segura, M Gutiérrez \& L Vásquez. 2004. From small-scale habitat loopholes to decadal cycles: a habitat-based hypothesis explaining fluctuation in pelagic fish populations off Peru. Fish and Fisheries 5: 296-316.

Bückle LF, B Barón, M Hernández, A Ledo, R Solís, B Pérez \& A Hernández. 2003. Sistema de temperatura oxígeno y salinidad para la experimentación en ecofisiología. Hidrobiológica 13: 277-287.

Butler JL, PE Smith \& CH Lo. 1993. The effect of natural variability of lie-history parameters on anchovy and sardine population growth. CalCOFI Reports 34: 104-111.

Clark FN \& JF Janssen. 1945. Movements and abundance of the sardine as measured by tag returns. California Department of Fish and Game, Fish Bulletin 61: 7-42.

Emmett RL, RD Brodeur, TW Miller, SS Pool, GK Krutzkowsky, PJ Bentley \& J Mc Crae. 2005. Pacific sardine (Sardinops sagax) abundance, distribution, and ecological relationships in the Pacific Northwest. CalCOFI Reports 46: 122-143.

Félix-Uraga R, VM Gómez-Muñoz, W García-Franco, FC Quiñonez-Velazquez \& FN Melo-Barrera. 2004. On the existence of Pacific sardine groups off the West coast of Baja California and southern California. CalCOFI Reports 45: 146-151.
Félix-Uraga R, VM Gómez-Muñoz, C Quiñonez-Velázquez, FN Melo-Barrera \& W García-Franco. 2005. Pacific sardine (Sardinops sagax) stock discrimination off the west coast of Baja California and southern California using otolith morphometry. CalCOFI Reports 46: 113-121.

Hernández-Rodríguez M, LF Bückle-Ramírez \& S EspinaAguilera. 2002. Temperature preference and acclimation in Poecilia sphenops (Pisces, Poeciliidae). Aquaculture Research 33: 933-940.

Lluch-Belda D, FJ Magallón \& RA Schwartzlose. 1986. Large fluctuations in the sardine fishery in the Gulf of California: Possible causes. CalCOFI Reports 27:136-140.

Lluch-Belda D, DB Lluch-Cota, S Hernández-Vázquez \& CA Salina-Zavala. 1991. Sardine and anchovy spawning as related to temperature and upwelling in the California Current system. CalCOFI Reports 32: 105-111.

Lynn RJ, KA Bliss \& LE Eber. 1982. Vertical and horizontal distributions of seasonal mean temperature, salinity, sigmat, stability, dynamic height, oxygen, and oxygen saturation in the California current, 1950-1978. CalCOFI Atlas 30: 1-530.

Martinez-Porchas M. 2009. Comportamiento termorregulador y respuesta de los parámetros sanguíneos de Sardinops sagax caeruleus a diferentes fluctuaciones térmicas. Tesis de Doctorado, Departamento de Acuicultura, CICESE, Ensenada, 108 pp.

Martínez-Porchas M, M Hernández-Rodríguez \& LF Bückle-Ramírez. 2009. Thermal behavior of the Pacific sardine (Sardinops sagax) acclimated to different thermal cycles. Journal of Thermal Biology 34: 372-376.

Santos AMP, A Dos Santos \& A Peliz. 2006. Vertical distribution of the European sardine (Sardina pilchardus) larvae and its implications for their survival. Journal of Plankton Research 28: 253-532.

Takasuka A, Y Oozeki \& I Aoki. 2007. Optimal growth temperature hypothesis: Why do anchovy flourish and sardine collapse or vice versa under the same ocean regime? Canadian Journal of Fisheries and Aquatic Sciences 64: 768-776.

Zar JH. 1996. Biostatistical analysis, 662 pp. Prentice-Hall, Upper Saddle River.

Recibido el 8 de febrero de 2010 y aceptado el 29 de julio de 2010 